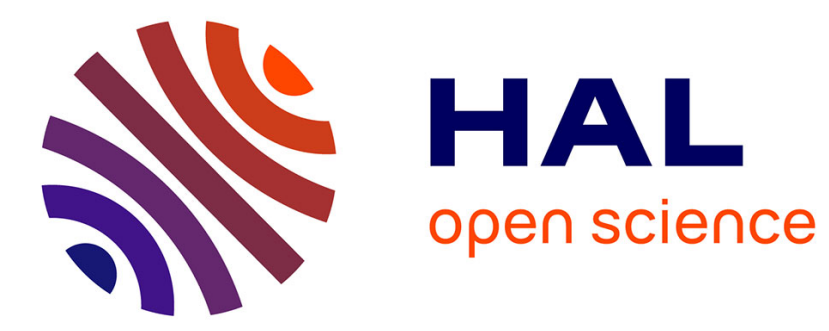

\title{
Spin Geometry and Image Processing
}

\author{
Michel Berthier
}

\section{To cite this version:}

Michel Berthier. Spin Geometry and Image Processing. 2013. hal-00801224

\section{HAL Id: hal-00801224 \\ https://hal.science/hal-00801224}

Preprint submitted on 15 Mar 2013

HAL is a multi-disciplinary open access archive for the deposit and dissemination of scientific research documents, whether they are published or not. The documents may come from teaching and research institutions in France or abroad, or from public or private research centers.
L'archive ouverte pluridisciplinaire HAL, est destinée au dépôt et à la diffusion de documents scientifiques de niveau recherche, publiés ou non, émanant des établissements d'enseignement et de recherche français ou étrangers, des laboratoires publics ou privés. 


\title{
Spin Geometry and Image Processing
}

\author{
Michel Berthier
}

\begin{abstract}
We give a survey of applications of spin geometry to image processing. We mainly focus on the problem of defining geometric Fourier transforms for both grey-level and color images. The definitions we propose rely on a spin generalization of the usual notion of character. We consider three possibilities for the actions of these spin characters: by using the spinor representation of grey-level image surfaces; by considering grey-level and color images as sections of associated bundles built first with standard representations and then with spin representations. Examples of applications to low-pass filtering are presented.
\end{abstract}

Mathematics Subject Classification (2010). Primary 68U10, 53C27; Secondary 53A05, 43A32.

Keywords. Spin Geometry, Image Processing, Clifford Fourier Transform.

\section{Introduction}

In this paper, we mainly adress the following question: how to define a Fourier transform for color image processing, which does not reduce to three Fourier transforms computed marginally, that accounts for color data and for the geometry of the underlying Riemannian image surface?

This question is motivated for instance by the well known fact that marginal processings produce false colors and thus are not suitable for signal synthesis (see for instance [25]). It has received increasing attention since the seminal work of S. Sangwine and co-authors whose original idea was to embed the acquisition space of RGB color channels into the 3-dimensional vector space of imaginary quaternions ([17], [29]). Lots of works are nowadays devoted to the more general question of defining Fourier transforms in the framework of Clifford algebras (see for instance [4], [6], [7], [8] [9], [10], [11], [14], [15], [16], [18], [19]). In this contribution, we adress this issue from the viewpoint of group actions and representations which are the basic notions of the abstract Fourier transform theory ([32]). We are led to consider group morphisms from the abelian group $\left(\mathbb{R}^{2},+\right)$ into spin groups which are called 
spin characters. One important fact is that these spin characters, if they are as usual parametrized by frequencies, are also parametrized by bivectors of the involved Clifford algebra.

From a geometrical viewpoint a $n$-dimensional image can be considered as a surface $\Sigma$ embedded in $\mathbb{R}^{n+2}$ by the global chart

$$
\left(x_{1}, x_{2}\right) \in \Omega \longmapsto\left(x_{1}, x_{2}, f_{1}\left(x_{1}, x_{2}\right), \ldots, f_{n}\left(x_{1}, x_{2}\right)\right)
$$

where $\Omega$ denotes the image domain. This surface inherits a Riemannian metric by restricting the Euclidean scalar product of $\mathbb{R}^{n+2}$ on each tangent space. It is well known that strong variations of this metric correspond to edges in the image (see for instance [1] and [5]). One key idea here is to involve the variations of the metric in the definition of the Fourier transform. For this, we take advantage of the fact that one can use the bivectors incoding the tangent planes of $\Sigma$ as parameters of the above spin characters.

Let us recall that spin groups admit two types of representations. The first ones are called standard representations. They describe spin groups as double-sheeted coverings of special orthogonal groups (see [3] or [28]). Beside these standard representations exist other representations called spin representations. These latter are restriction to spin groups of complex representations of Clifford algebras. They do not descend to orthogonal groups.

In the following we consider three possibilities for the actions of spin characters. The first one relies on the generalized Weierstrass parametrization of immersed surfaces of the Euclidean space $\mathbb{R}^{3}$ introduced by T. Friedrich (see [21] and Sec. 3). In this case the spin characters act through a spin representation on the spinor field that encodes the unit normal to a grey-level image surface (see [2]). This approach can not be generalized straightforwardly to color images since there exist no simple analog of the generalized Weierstrass parametrization for surfaces immersed in $\mathbb{R}^{n}$ for $n>4$.

To tackle this problem, we consider in Sec. 4 grey-level and color images as sections of associated bundles. These bundles are built using standard representations (see [3]). Finally, we study in Sec. 5 the case of spin representations, that is the case where images are seen as sections of spinor bundles.

We propose applications to low-pass filterings in each of these three cases. We give sometimes interpretations in terms of diffusion.

\section{Mathematical Background}

We introduce in this section the mathematical notions and results needed for the constructions. The reader may refer to [12], [20], [23], [24], [27], [28] and [32] for further details and explanations.

\subsection{Abstract Fourier Transform, Spin Groups, Representations and Associ- ated Bundles}

The Pontryagin dual $\widehat{G}$ of a locally compact unimodular group $G$ is the set of equivalence classes of irreducible unitary representations of $G$. The Fourier 
transform of a function $f$ in $L^{2}(G, \mathbb{C})$ is defined on $\widehat{G}$ by

$$
\mathcal{F}(f)(\varphi)=\int_{G} f(g) \varphi\left(g^{-1}\right) d \nu(g)
$$

where $\nu$ is a Haar measure on $G$. In what follows we only consider the case where $G=\left(\mathbb{R}^{2},+\right)$ (although more general cases have been already envisaged in image processing, see for instance [13] and [30]). Since $G$ is abelian, every irreducible representation is of dimension one and is given by a continuous group morphism

$$
\varphi_{u_{1}, u_{2}}:\left(x_{1}, x_{2}\right) \longmapsto \exp \left[2 i \pi\left(x_{1} u_{1}+x_{2} u_{2}\right)\right]
$$

from the group $\left(\mathbb{R}^{2},+\right)$ to the group $\mathbb{S}^{1}$ of unit complex numbers. Such a morphism is called a character of $G$. Applying the above formula (2.1) leads to the usual definition of the Fourier transform of a complex valued function $f$ defined on $\mathbb{R}^{2}$. The discrete version provides a decomposition

$$
f(m, n)=\sum_{u_{1}, u_{2}} \widehat{f}\left(u_{1}, u_{2}\right) \exp \left[2 i \pi\left(u_{1} m / M+u_{2} n / N\right)\right]
$$

for a grey-level image $f$ where

$$
\widehat{f}\left(u_{1}, u_{2}\right)=\sum_{m, n} f(m, n) \exp \left[-2 i \pi\left(u_{1} m / M+u_{2} n / N\right)\right]
$$

is the 2-dimensional discrete Fourier transform. As mentioned in the introduction this decomposition doesn't account for the geometry of the image graph.

It is well known that $\mathbb{S}^{1}$ is isomorphic to the group $\operatorname{Spin}(2)$ and one key idea of what follows consists in replacing in the definition of character the group $\mathbb{S}^{1}$ by the group $\operatorname{Spin}(n)$. Let us first recall that the standard representation of $\operatorname{Spin}(n)$ is given by the group morphism

$$
\rho_{n}: \operatorname{Spin}(n) \longrightarrow S O(n, \mathbb{R})
$$

with

$$
\rho_{n}(\tau): u \longrightarrow u \perp \tau:=\tau u \tau^{-1}
$$

The morphism $\rho_{n}$ is a 2 -sheeted covering, its kernel being isomorphic to $\mathbb{Z} / 2 \mathbb{Z}$. In Sec. 4 , we will consider the cases $n=4$ and $n=6$.

The spin representations of $\operatorname{Spin}(n)$ are obtained from the complex representations of the corresponding complex Clifford algebras. They do not descend to the orthonormal groups. In the sequel (Sec. 3 and Sec. 5), we only consider the spin representation of $\operatorname{Spin}(3)$. It is obtained as the restriction to $\operatorname{Spin}(3)$ of an irreducible complex representation of the algebra $\mathbb{C}(2) \oplus \mathbb{C}(2)$, the space of representation being $\mathbb{C}^{2}$ (see [2] for the explicit definition). We will denote

$$
\zeta_{3}: \operatorname{Spin}(3) \longrightarrow \mathbb{C}(2)
$$

this spin representation.

Let $\Omega$ be a domain of $\mathbb{R}^{2}$ (the image domain) and consider the trivial bundle $E_{n}(\Omega)=\Omega \times \mathbb{R}^{n}$. A spin structure is given by a 2 -sheeted covering

$$
P_{\text {Spin }}\left(E_{n}(\Omega)\right) \longmapsto P_{S O}\left(E_{n}(\Omega)\right)
$$


compatible with $\operatorname{Spin}(n)$ and $S O(n, \mathbb{R})$ actions. Here $P_{S O}\left(E_{n}(\Omega)\right)$ denotes the $S O(n, \mathbb{R})$-principal bundle of direct frames. Using the representations $\rho_{n}$ and $\zeta_{3}$, one can define the associated bundles

$$
\Delta_{\rho_{n}}(\Omega)=P_{\text {Spin }}\left(E_{n}(\Omega)\right) \times_{\rho_{n}} \mathbb{R}^{n}
$$

and

$$
\Delta_{\zeta_{3}}(\Omega)=P_{\text {Spin }}\left(E_{3}(\Omega)\right) \times_{\zeta_{3}} \mathbb{C}^{2}
$$

\subsection{Spin Characters}

As mentioned before, the idea to define a Fourier transform that is suitable for frequency analysis of $n$-dimensional images is to replace the usual characters of $\left(\mathbb{R}^{2},+\right)$ by so-called spin characters.

Definition 2.1. A $\operatorname{Spin}(n)$ character of $\mathbb{R}^{2}$ is a Lie group morphism from $\mathbb{R}^{2}$ to $\operatorname{Spin}(n)$.

Since $\mathbb{R}^{2}$ is simply connected, the $\operatorname{Spin}(n)$ characters of $\mathbb{R}^{2}$ are given by exponentiating Lie algebra homomorphisms from $\mathbb{R}^{2}$ (the Lie algebra of the group $\mathbb{R}^{2}$ ) to the Lie algebra $\mathfrak{s p i n}(n)$ of $\operatorname{Spin}(n)$. We consider the cases $n=3, n=4$ and $n=6$.

Theorem 2.2 (Spin(3) characters). The Spin(3) characters are given by

$$
\left(x_{1}, x_{2}\right) \longmapsto \exp \frac{1}{2}\left[B A\left(\begin{array}{l}
x_{1} \\
x_{2}
\end{array}\right)\right]=\exp \frac{1}{2}\left[\left(x_{1} u_{1}+x_{2} u_{2}\right) B\right]
$$

where

$$
A=\left(\begin{array}{l}
u_{1} \\
u_{2}
\end{array}\right) \text { and } B=e f
$$

with $u_{1}$ and $u_{2}$ real numbers (the frequencies) and e and $f$ two orthonormal vectors of $\mathbb{R}^{3}$.

Theorem 2.3 (Spin(4) and Spin(6) characters). The Spin( $n)$ characters, $n=$ 4,6 , are given by

$$
\left(x_{1}, x_{2}\right) \longmapsto \exp \frac{1}{2}\left[\left(B_{1} \cdots B_{n / 2}\right) A_{n / 2}\left(\begin{array}{c}
x_{1} \\
x_{2}
\end{array}\right)\right]
$$

where $A_{n / 2}$ is a $(n / 2) \times 2$ matrix with real coeffcients (the frequencies),

$$
B_{i}=e_{i} f_{i}
$$

for $i=1, \ldots, n / 2$ and $\left(e_{1}, \ldots, e_{n / 2}, f_{1}, \ldots, f_{n / 2}\right)$ denotes an orthonormal basis of $\mathbb{R}^{n}$.

The proofs in the cases $n=3$ and $n=4$ can be found in [4]. They rely on the facts that the abelian Lie subalgebras of $\mathfrak{s p i n}(3)$ are of dimension 1 and that $\operatorname{Spin}(4)$ is isomorphic to $\operatorname{Spin}(3) \times \operatorname{Spin}(3)$. The case $n=6$ is treated in [3] using the isomorphism $\operatorname{Spin}(6) \simeq S U(4)$. Note that these results are closely related to D. Hestenes orthogonalization algorithm for bivectors (see [22]). In the following we denote $\chi_{A, B}$ these spin characters where $B$ stands for $B$ (for $n=3),\left(B_{1} B_{2}\right)$ (for $\left.n=4\right)$ or $\left(B_{1} B_{2} B_{3}\right)$ (for $\left.n=6\right)$, depending on the context. 
It appears that the spin characters are parametrized by frequencies (the entries of the matrix $A$ ) and by a set $B$ of othonormal bivectors.

\subsection{Spinor Representation of Surfaces Following T. Friedrich}

The spinor representation of surfaces introduced in [21] generalizes the Weierstrass representation of minimal surfaces.

Theorem 2.4 (T. Friedrich spinor representation of surfaces). There is a one-to-one correspondance between spinor fields $\varphi^{*}$ of constant length on a Riemannian surface $(\Sigma, g)$ and satisfying

$$
D \varphi^{*}=H \varphi^{*}
$$

where $D$ is the Dirac operator in one hand, and isometric immersions of $\Sigma$ in $\mathbb{R}^{3}$ with mean curvature equal to $H$, on the other hand.

Let us recall briefly how to obtain $\varphi^{*}$. Let $\nu$ be the unit normal to the surface $\Sigma$ and $\phi$ be a parallel spinor field of the Euclidean space $\mathbb{R}^{3}$. The restriction $\varphi=\phi_{\mid \Sigma}$ decomposes into $\varphi=\varphi^{+}+\varphi^{-}$where

$$
\varphi^{+}=\frac{1}{2}(\varphi+i \nu \cdot \varphi) \text { and } \varphi^{-}=\frac{1}{2}(\varphi-i \nu \cdot \varphi)
$$

and satisfies

$$
D \varphi=-H \cdot \nu \cdot \varphi
$$

that is

$$
D\left(\varphi^{+}+\varphi^{-}\right)=-H \cdot \nu \cdot\left(\varphi^{+}+\varphi^{-}\right)
$$

This implies

$$
D \varphi^{+}=-i H \varphi^{-} \text {and } D \varphi^{-}=i H \varphi^{+}
$$

The spinor field $\varphi^{*}=\varphi^{+}-i \varphi^{-}$satisfies equation (2.15) and is of constant length.

Since the spinor field $\varphi^{*}$ encodes all the geometry of the immersed surface $(\Sigma, g)$, it may be useful to perform image processing of grey-level images. In the next section we propose to deal with Fourier transform of such spinor fields.

\section{Spinor Fourier Transform with Spinor Surface Representations ([2])}

We consider a grey-level image as a surface $(\Sigma, g)$ immersed in $\mathbb{R}^{3}$ by the parametrization

$$
\left(x_{1}, x_{2}\right) \in \Omega \longmapsto\left(x_{1}, x_{2}, f\left(x_{1}, x_{2}\right)\right)
$$

where $\Omega$ is the image domain. 


\subsection{Spinor Representation of Grey-Level Images}

The unit normal $\nu$ of the surface $\Sigma$ is given by

$$
\nu=\frac{1}{\Delta}\left(-f_{x_{1}} e_{1}-f_{x_{2}} e_{2}+e_{3}\right)
$$

where

$$
\Delta=\sqrt{f_{x_{1}}^{2}+f_{x_{2}}^{2}+1}
$$

and $f_{x_{i}}$ denotes the partial derivative of $f$ with respect to $x_{i}$.

Proposition 3.1. The spinor field $\varphi^{*}$ is given by

$$
\varphi^{*}=\frac{1}{2}(1-i)\left(\begin{array}{c}
\left(1-\frac{i f_{x_{2}}}{\Delta}\right) u+\left(\frac{1+i f_{x_{1}}}{\Delta}\right) v \\
\left(1+\frac{i f_{x_{2}}}{\Delta}\right) v+\left(\frac{i f_{x_{1}}-1}{\Delta}\right) u
\end{array}\right)
$$

where $u$ and $v$ are complex numbers.

The proof is given in [2]. Let us just mention that the complex numbers $u$ and $v$ are the components of the parallel spinor $\phi$ mentioned above. To determine them one integrates period forms to recover the original immersion so as to obtain: $u=1 / \sqrt{2}$ and $v=-1 / \sqrt{2}$. The spinor field $\varphi^{*}$ is actually given by

$$
\varphi^{*}=\frac{1}{2 \sqrt{2}}(1-i)\left(\begin{array}{c}
1-\frac{1+i\left(f_{x_{1}}+f_{x_{2}}\right)}{\Delta} \\
-\left(1+\frac{1+i\left(-f_{x_{1}}+f_{x_{2}}\right)}{\Delta}\right)
\end{array}\right)
$$

\subsection{Fourier Transform of spinor fields}

We deal here with $\operatorname{Spin}(3)$ characters:

$$
\chi_{A, B}(A)=\exp \left[\frac{1}{2}\left(x_{1} u_{1}+x_{2} u_{2}\right) B\right]
$$

where

$$
A=\left(\begin{array}{l}
u_{1} \\
u_{2}
\end{array}\right)
$$

Recall that the group $\operatorname{Spin}(3)$ acts on the sections of the spinor bundle $S(\Sigma)$ of the surface $\Sigma$ (the action is denoted $\cdot$ ).

Definition 3.2 (Fourier transform of spinor fields). The Fourier transform of a spinor field $\varphi$ is given by

$$
\mathcal{F}(\varphi)\left(u_{1}, u_{2}\right)=\int_{\mathbb{R}^{2}} \chi_{u_{1}, u_{2}, z_{1} \wedge z_{2}\left(x_{1}, x_{2}\right)}\left(-x_{1},-x_{2}\right) \cdot \varphi\left(x_{1}, x_{2}\right) d x_{1} d x_{2}
$$

where $\left(z_{1}, z_{2}\right)$ is an orthonormal frame of the tangent bundle $T(\Sigma)$ and $z_{1} \wedge$ $z_{2}\left(x_{1}, x_{2}\right)$ is the corresponding bivector at the point $\left(x_{1}, x_{2}\right)$. 
This definition is in fact a particular case (involving the tangent bivector $z_{1} \wedge z_{2}$ ) of a more general definition that could be stated with an arbitrary bivector field $B\left(x_{1}, x_{2}\right)$ of $\mathbb{R}_{3,0}$.

The two eigenvalues of the operator defined by the Clifford action of $z_{1} \wedge z_{2}$ on the spinor bundle $S(\Sigma)$ are $i$ and $-i$. Let $\gamma_{i}$ and $\gamma_{-i}$ be corresponding unit eigenspinor fields. We have

$$
\begin{gathered}
\chi_{u_{1}, u_{2}, z_{1} \wedge z_{2}\left(x_{1}, x_{2}\right)}\left(-x_{1},-x_{2}\right) \cdot \varphi\left(x_{1}, x_{2}\right)= \\
e^{i / 2\left(u_{1} x_{1}+u_{2} x_{2}\right)} \varphi^{+}\left(x_{1}, x_{2}\right) \gamma_{-i}\left(x_{1}, x_{2}\right)+e^{-i / 2\left(u_{1} x_{1}+x_{2} x_{2}\right)} \varphi^{-}\left(x_{1}, x_{2}\right) \gamma_{i}\left(x_{1}, x_{2}\right)
\end{gathered}
$$

where $\varphi^{+}$, respectively $\varphi^{-}$, is the projection of $\varphi$ on the eigenspace corresponding to the eigenvalue $i$, respectively $-i$. The formula (3.8) can be rewritten in the frame $\left(\gamma_{-i}, \gamma_{i}\right)$

$$
\mathcal{F}(\varphi)\left(u_{1}, u_{2}\right)=\left({\widehat{\varphi^{+}}}^{-1}\left(u_{1}, u_{2}\right), \widehat{\varphi^{-}}\left(u_{1}, u_{2}\right)\right)
$$

where ${ }^{\widehat{ }}$ and ${ }^{\widehat{-1}}$ denote the Fourier transform and its iverse.

As a consequence the Fourier transform of a spinor field can be computed with usual fast Fourier transforms.

\subsection{Applications}

We give here an example of how to use the above defined Fourier transform for spinor fields in the context of grey-level image processing. We use here a discrete version of the above definition (this will be also tacitly the case for the applications in Sec. 4 and Sec. 5). Every spinor field $\varphi$ can be written as

$$
\varphi=\sum_{m, n} \varphi_{m, n}
$$

with

$$
\varphi_{m, n}\left(u_{1}, u_{2}\right)=\rho_{u_{1}, u_{2}, z_{1} \wedge z_{2}(m, n)}(m, n) \cdot \mathcal{F}(\varphi)(m, n)
$$

If we write

$$
\varphi_{m, n}=\left(\varphi_{m, n}^{+}, \varphi_{m, n}^{-}\right)
$$

in the frame $\left(\gamma_{-i}, \gamma_{i}\right)$, then the components of $\varphi_{m, n}$ are given by

$$
\begin{gathered}
\varphi_{m, n}^{+}\left(u_{1}, u_{2}\right)=e^{-2 \pi i\left(u_{1} m / M+u_{2} n / N\right)}{\widehat{\varphi^{+}}}^{-1}(m, n) \\
\varphi_{m, n}^{-}\left(u_{1}, u_{2}\right)=e^{2 \pi i\left(u_{1} m / M+u_{2} n / N\right)} \widehat{\varphi^{-}}(m, n)
\end{gathered}
$$

and satisfy

$$
\left|\varphi_{m, n}\right|^{2}=\left|\varphi_{m, n}^{+}\right|^{2}+\left|\varphi_{m, n}^{-}\right|^{2}
$$

Let now $f$ be a grey-level image and let $\varphi^{*}$ be the corresponding spinor field obtained from the spinor representation of the image graph of $f$. To perform the filtering, we apply a Gaussian mask $T_{\sigma}$ of variance $\sigma$ in the spectrum $\mathcal{F}\left(\varphi^{*}\right)$ of $\varphi^{*}$. The resulting image is given by the function

$$
\tilde{f}=\left|\mathcal{F}^{-1} T_{\sigma} \mathcal{F} \varphi^{*}\right| f
$$

Fig. 1 shows results of such low-pass filterings. 


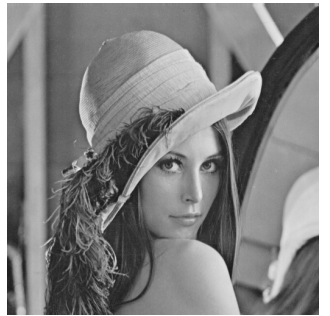

(a) Original

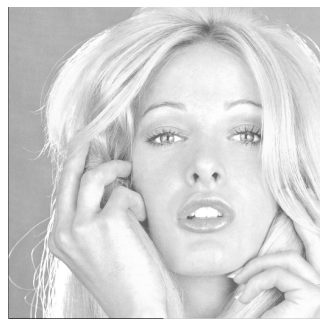

(c) Original

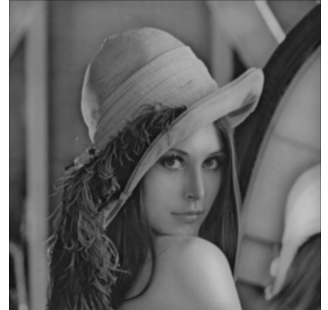

(b) $\sigma=100$

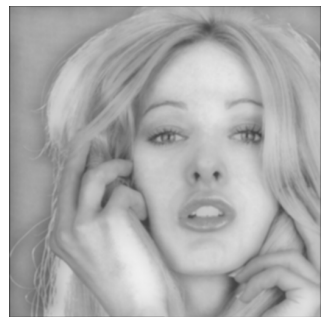

(d) $\sigma=100$

FiguRE 1. Low-pass filtering using $\left|\mathcal{F}^{-1} T_{\sigma} \mathcal{F} \varphi^{*}\right| f$

\subsection{Remarks}

Generalizing the above construction to color images is not straightforward. This is due to the fact that there do not exist generalized Weierstrass parametrization for surfaces embedded in $\mathbb{R}^{n}, n>4$. The only Grassmannians $G_{n, 2}$ of 2-planes of $\mathbb{R}^{n}$ that are rational are $G_{3,2}$ (isomorphic to $\mathbb{C} P^{1}$ ) and $G_{4,2}$ (isomorphic to $\mathbb{C} P^{1} \times \mathbb{C} P^{1}$ ), see [31]. The aim of the next sections is to show how to tackle this problem by considering images themselves as sections of associated bundles.

\section{Spinor Fourier Transform for Images using Standard Representations ([3])}

In this section, we consider images as sections of associated bundles built with the standard representations

$$
\rho_{n}: \operatorname{Spin}(n) \longrightarrow S O(n, \mathbb{R})
$$

for $n=4$ (grey-level images) and $n=6$ (color images).

\subsection{Images as Sections of Associated Bundles}

Let

$$
f:\left(x_{1}, x_{2}\right) \in \Omega \longmapsto f\left(x_{1}, x_{2}\right)
$$


be a grey-level image. Such an image is considered as a section of the associated bundle $P_{S p i n}\left(E_{4}(\Omega)\right) \times_{\rho_{4}} \mathbb{R}^{4}$. More precisely, to $f$ corresponds a map

$$
\sigma_{f}: \Omega \longrightarrow P_{S p i n}\left(E_{4}(\Omega)\right) \times_{\rho_{4}} \mathbb{R}^{4}
$$

that satisfies

$$
\pi_{4} \circ \sigma_{f}=I d
$$

where

$$
\pi_{4}: P_{S p i n}\left(E_{4}(\Omega)\right) \times_{\rho_{4}} \mathbb{R}^{4} \longrightarrow \mathbb{R}^{4}
$$

is the vector bundle projection. This section is explicitely given by

$$
\sigma_{f}\left(x_{1}, x_{2}\right)=f\left(x_{1}, x_{2}\right) e_{3}
$$

When dealing with a color image

$$
f:\left(x_{1}, x_{2}\right) \in \Omega \longmapsto\left(f_{1}\left(x_{1}, x_{2}\right), f_{2}\left(x_{1}, x_{2}\right), f_{3}\left(x_{1}, x_{2}\right)\right)
$$

we consider the section

$$
\sigma_{f}\left(x_{1}, x_{2}\right)=f_{1}\left(x_{1}, x_{2}\right) e_{3}+f_{2}\left(x_{1}, x_{2}\right) e_{4}+f_{3}\left(x_{1}, x_{2}\right) e_{5}
$$

of the associated vector bundle $P_{S p i n}\left(E_{6}(\Omega)\right) \times{ }_{\rho_{6}} \mathbb{R}^{6}$. In both cases, at a given point $\left(x_{1}, x_{2}\right)$ of $\Omega$ the value of the section $\sigma_{f}$ belongs to a representation space on which the spin group considered as the fiber of $P_{S p i n}\left(E_{n}(\Omega)\right)$ at $\left(x_{1}, x_{2}\right)$ acts. This action allows to consider varying spin characters.

For a grey-level image

$$
f:\left(x_{1}, x_{2}\right) \in \Omega \longmapsto f\left(x_{1}, x_{2}\right)
$$

we consider the surface $\Sigma_{f}$ embedded in $\mathbb{R}^{3}$ by the parametrization

$$
\varphi:\left(x_{1}, x_{2}\right) \longmapsto\left(x_{1}, x_{2}, f\left(x_{1}, x_{2}\right)\right)
$$

that is the 2-dimensional Riemannian surface with a global chart $(\Omega, \varphi)$. The Riemannian metric on $\Sigma_{f}$ is the one induced by the Euclidean metric of $\mathbb{R}^{3}$. In the same way, if

$$
f:\left(x_{1}, x_{2}\right) \in \Omega \longmapsto\left(f_{1}\left(x_{1}, x_{2}\right), f_{2}\left(x_{1}, x_{2}\right), f_{3}\left(x_{1}, x_{2}\right)\right)
$$

is a color image, it can be considered as a surface $\Sigma_{f}$ embedded in $\mathbb{R}^{5}$ by the parametrization

$$
\varphi:\left(x_{1}, x_{2}\right) \mapsto\left(x_{1}, x_{2},\left(f_{1}\left(x_{1}, x_{2}\right), f_{2}\left(x_{1}, x_{2}\right), f_{3}\left(x_{1}, x_{2}\right)\right)\right.
$$

and in this case the Riemannian metric is the one induced by $\mathbb{R}^{5}$.

The idea in what follows consists in using the bivector field encoding the tangent planes of $\Sigma_{f}$ as a parametrizing bivector field for the spin characters. 


\subsection{Tangent Bundle and Bivector fields}

At each point $p$ of $\Omega$, the embedding space $\mathbb{R}^{3}$ of $\Sigma_{f}$, for $f$ a grey-level image, splits into

$$
\mathbb{R}^{3}=T_{\varphi(p)} \Sigma_{f} \oplus N_{\varphi(p)} \Sigma_{f}
$$

where $T \Sigma_{f}$, resp. $N \Sigma_{f}$, denotes the tangent, resp. the normal, bundle of the surface $\Sigma_{f}$. Let $F_{4}$ be the bundle

$$
F_{4}=T_{\Sigma} \oplus N \Sigma_{f} \oplus \mathbb{R} e_{4}
$$

and $C l\left(F_{4}\right)$ be the corresponding Clifford bundle. Identifying the degree one sections of $C l\left(F_{4}\right)$ with functions from $\Omega$ to $\mathbb{R}^{4}$ we can consider the image $f$ as a section $\sigma_{f}\left(x_{1}, x_{2}\right)=f\left(x_{1}, x_{2}\right) e_{3}$ of $C l\left(F_{4}\right)$. The tangent planes are encoded by the degree 2 section (the bivector field) of $C l\left(F_{4}\right)$

$$
B_{1}=\frac{\varphi_{x_{1}} \wedge \varphi_{x_{2}}}{\left\|\varphi_{x_{1}} \wedge \varphi_{x_{2}}\right\|}=\gamma_{1} e_{1} e_{2}+\gamma_{2} e_{1} e_{3}+\gamma_{3} e_{2} e_{3}
$$

where

$$
\gamma_{1}=\frac{1}{\sqrt{1+f_{x_{1}}^{2}+f_{x_{2}}^{2}}} \quad \gamma_{2}=\frac{f_{x_{2}}}{\sqrt{1+f_{x_{1}}^{2}+f_{x_{2}}^{2}}}
$$

and

$$
\gamma_{3}=\frac{-f_{x_{1}}}{\sqrt{1+f_{x_{1}}^{2}+f_{x_{2}}^{2}}}
$$

In this case $B_{2}$ is given by $I_{4} B_{1}$ where $I_{4}$ denotes the pseudoscalar of the Clifford algebra $\mathbb{R}_{4,0}$. We have

$$
B_{2}=-\gamma_{3} e_{1} e_{4}+\gamma_{2} e_{2} e_{4}-\gamma_{1} e_{3} e_{4}
$$

For a color image, we introduce the decomposition

$$
\mathbb{R}^{5}=T_{\varphi(p)} \Sigma_{f} \oplus N_{\varphi(p)} \Sigma_{f}
$$

the bundle

$$
F_{6}=T \Sigma_{f} \oplus N \Sigma_{f} \oplus \mathbb{R} e_{6}
$$

the Clifford bundle $C l\left(F_{6}\right)$ and identify the color image with a degree one section $\sigma_{f}\left(x_{1}, x_{2}\right)=f_{1}\left(x_{1}, x_{2}\right) e_{3}+f_{2}\left(x_{1}, x_{2}\right) e_{4}+f_{3}\left(x_{1}, x_{2}\right) e_{5}$ of $C l\left(F_{6}\right)$. The corresponding bivector $B_{1}$ is given by

$$
\begin{gathered}
B_{1}=\gamma_{1} e_{1} e_{2}+\gamma_{2} e_{1} e_{3}+\gamma_{3} e_{1} e_{4}+\gamma_{4} e_{1} e_{5}+\gamma_{5} e_{2} e_{3} \\
+\gamma_{6} e_{2} e_{4}+\gamma_{7} e_{2} e_{5}+\gamma_{8} e_{3} e_{4}+\gamma_{9} e_{3} e_{5}+\gamma_{10} e_{4} e_{5}
\end{gathered}
$$

where

$$
\begin{gathered}
\gamma_{1}=1 / \delta \gamma_{2}=\left(f_{1}\right)_{x_{2}} / \delta \gamma_{3}=\left(f_{2}\right)_{x_{2}} / \delta \gamma_{4}=\left(f_{3}\right)_{x_{2}} / \delta \\
\gamma_{5}=\left(-f_{1}\right)_{x_{1}} / \delta \gamma_{6}=\left(-f_{2}\right)_{x_{1}} / \delta \gamma_{7}=\left(-f_{3}\right)_{x_{1}} / \delta \\
\gamma_{8}=\left[\left(f_{1}\right)_{x_{1}}\left(f_{2}\right)_{x_{2}}-\left(f_{1}\right)_{x_{2}}\left(f_{2}\right)_{x_{1}}\right] / \delta \\
\gamma_{9}=\left[\left(f_{1}\right)_{x_{1}}\left(f_{3}\right)_{x_{2}}-\left(f_{1}\right)_{x_{2}}\left(f_{3}\right)_{x_{1}}\right] / \delta \\
\gamma_{10}=\left[\left(f_{2}\right)_{x_{1}}\left(f_{3}\right)_{x_{2}}-\left(f_{2}\right)_{x_{2}}\left(f_{3}\right)_{x_{1}}\right] / \delta
\end{gathered}
$$

with $\delta=\left\|\varphi_{x_{1}} \wedge \varphi_{x_{2}}\right\|$. 


\subsection{Spinor Fourier Transform using Standard Representations}

Let $f$ be a grey-level or color image and $\sigma_{f}$ be the corresponding section of the bundle $P_{\text {Spin }}\left(E_{n}(\Omega)\right) \times_{\rho_{n}} \mathbb{R}^{n}, n=4$ or $n=6$. We introduce

$$
\left.\widetilde{\sigma}\left(x_{1}, x_{2}, y_{1}, y_{2}\right)=\sum_{i} \delta_{(} y_{1}, y_{2}\right) e_{i}\left(x_{1}, x_{2}\right)+\sum_{i} \tau_{i}\left(y_{1}, y_{2}\right) f_{i}\left(x_{1}, x_{2}\right)
$$

where

$$
\delta_{i}\left(y_{1}, y_{2}\right)=\sigma\left(y_{1}, y_{2}\right) \cdot e_{i}\left(y_{1}, y_{2}\right) \quad \tau_{i}\left(y_{1}, y_{2}\right)=\sigma\left(y_{1}, y_{2}\right) \cdot f_{i}\left(y_{1}, y_{2}\right)
$$

Definition 4.1 (Spinor Fourier transform - Standard Representations). With the previous notations, the spinor Fourier transform of $\sigma_{f}$ is given by

$$
\mathcal{F}_{B} \sigma_{f}(A)=\int_{\mathbb{R}^{2}} \tilde{\sigma}_{f}\left(x_{1}, x_{2}, y_{1}, y_{2}\right) \perp \chi_{A, B\left(x_{1}, x_{2}\right)}\left(-y_{1},-y_{2}\right) d y_{1} d y_{2}
$$

where $B_{1}=B_{1}\left(x_{1}, x_{2}\right)$ is given by (4.15) or (4.21) and $B=B\left(x_{1}, x_{2}\right)$ satisfies for each $\left(x_{1}, x_{2}\right)$ of $\Omega$ the conditions (2.14).

This spinor Fourier transform for a grey-level image is computed as follows:

$$
\begin{gathered}
\mathcal{F}_{B} \sigma_{f}\left(u_{1}, v_{1}, u_{2}, v_{2}\right)= \\
\int_{\mathbb{R}^{2}} e^{-\left(u_{1} y_{1}+v_{1} y_{2}\right) B_{1}\left(x_{1}, x_{2}\right)}\left[\delta_{1}\left(y_{1}, y_{2}\right) e_{1}\left(x_{1}, x_{2}\right)+\tau_{1}\left(y_{1}, y_{2}\right) f_{1}\left(x_{1}, x_{2}\right)\right] d y_{1} d y_{2}+ \\
\int_{\mathbb{R}^{2}} e^{-\left(u_{2} y_{1}+v_{2} y_{2}\right) B_{2}\left(x_{1}, x_{2}\right)}\left[\delta_{2}\left(y_{1}, y_{2}\right) e_{2}\left(x_{1}, x_{2}\right)+\tau_{2}\left(y_{1}, y_{2}\right) f_{2}\left(x_{1}, x_{2}\right)\right] d y_{1} d y_{2}
\end{gathered}
$$

Since $B_{i}\left(x_{1}, x_{2}\right)^{2}=-1$, we identify each one of the integrals

$$
\int_{\mathbb{R}^{2}} e^{-\left(u_{i} y_{1}+v_{i} y_{2}\right) B_{i}\left(x_{1}, x_{2}\right)}\left[\delta_{i}\left(y_{1}, y_{2}\right) e_{i}\left(x_{1}, x_{2}\right)+\tau_{i}\left(y_{1}, y_{2}\right) f_{i}\left(x_{1}, x_{2}\right)\right] d y_{1} d y_{2}
$$

for $i=1,2$, with the usual complex Fourier transform

$$
\int_{\mathbb{R}^{2}} e^{-\left(u_{i} y_{1}+v_{i} y_{2}\right) \sqrt{-1}}\left[\delta_{i}\left(y_{1}, y_{2}\right)+\sqrt{-1} \tau_{i}\left(y_{1}, y_{2}\right) d y_{1} d y_{2}\right]
$$

so that the result of the integration doesn't depend on $\left(x_{1}, x_{2}\right)$.

This means that

$$
\mathcal{F}_{B} \sigma_{f}\left(u_{1}, v_{1}, u_{2}, v_{2}\right)=
$$

reads

$$
\begin{gathered}
\int_{\mathbb{R}^{2}} e^{-\left(u_{1} y_{1}+v_{1} y_{2}\right) B_{1}\left(x_{1}, x_{2}\right)} f_{B_{1}\left(x_{1}, x_{2}\right)}\left(y_{1}, y_{2}\right) d y_{1} d y_{2}+ \\
\int_{\mathbb{R}^{2}} e^{-\left(u_{2} y_{1}+v_{2} y_{2}\right) B_{2}\left(x_{1}, x_{2}\right)} f_{B_{2}\left(x_{1}, x_{2}\right)}\left(y_{1}, y_{2}\right) d y_{1} d y_{2}
\end{gathered}
$$

where, for $i=1,2$,

$$
f_{B_{i}\left(x_{1}, x_{2}\right)}\left(y_{1}, y_{2}\right)=\delta_{i}\left(y_{1}, y_{2}\right) e_{i}\left(x_{1}, x_{2}\right)+\tau_{i}\left(y_{1}, y_{2}\right) f_{i}\left(x_{1}, x_{2}\right)
$$

is the projection of the section on the $B_{i}\left(x_{1}, x_{2}\right)$-plane. This shows in particular that the spinor Fourier transform can be computed using usual complex fast Fourier transforms. Note that an orthonormal frame field adapted to the 
$B_{1}$ and $B_{2}$-planes has to be introduced first. The spinor Fourier transform is invertible. More precisely:

$$
\begin{gathered}
\sigma_{f}\left(y_{1}, y_{2}\right)= \\
\int_{\mathbb{R}^{2}} e^{\left(u_{1} y_{1}+v_{1} y_{2}\right) B_{1}\left(x_{1}, x_{2}\right)} \mathcal{F}_{B_{1}}\left(f_{B_{1}}\right)\left(u_{1}, v_{1}\right) d u_{1} d v_{1}+ \\
\int_{\mathbb{R}^{2}} e^{\left(u_{2} y_{1}+v_{2} y_{2}\right) B_{2}\left(x_{1}, x_{2}\right)} \mathcal{F}_{B_{2}}\left(f_{B_{2}}\right)\left(u_{2}, v_{2}\right) d u_{2} d v_{2}
\end{gathered}
$$

where

$$
\begin{gathered}
\mathcal{F}_{B_{i}}\left(f_{B_{i}}\right)\left(u_{i}, v_{i}\right)= \\
\int_{\mathbb{R}^{2}} e^{-\left(u_{i} y_{1}+v_{i} y_{2}\right) B_{i}\left(x_{1}, x_{2}\right)} f_{B_{i}\left(x_{1}, x_{2}\right)}\left(y_{1}, y_{2}\right) d y_{1} d y_{2}
\end{gathered}
$$

for $i=1,2$. The procedure is the same for a color image.

\subsection{Applications}

Let $\sigma_{0}$ be a section corresponding to a grey-level image and $\left(\nu_{11}, \nu_{12}, \nu_{21}, \nu_{22}\right)$ be the frame field adapted to $B_{1}$ and $B_{2}=I_{4} B_{1}$ where $B_{1}$ encodes the tangent plane of the image surface. With the notations introduced above, we consider the equations

$$
\frac{\partial f_{B_{i}}}{\partial t_{i}}=\Delta_{B_{i}} f_{B_{i}}, \quad f_{B_{i} \mid t_{i}=0}=f_{0, B_{i}}
$$

for $i=1,2$. where $\Delta_{B_{i}}$ is the restriction of the Euclidean Laplacian $H$ in the frame field $\left(\nu_{11}, \nu_{12}, \nu_{21}, \nu_{22}\right)$. The solutions are given by

$$
f_{B_{i}}\left(\cdot, \cdot, t_{i}\right)=f_{0, B_{i}} * K_{t_{i}}
$$

where $K_{t_{i}}, i=1,2$, denote the Gaussian kernels. The system (4.33) is solved by

$$
f\left(\cdot, \cdot, t_{1}, t_{2}\right)=\mathcal{F}_{B}^{-1}\left(\mathcal{F}_{B_{1}} f_{0} \times \mathcal{F} K_{t_{1}}+\mathcal{F}_{B_{2}} f_{0} \times \mathcal{F} K_{t_{2}}\right)
$$

The heat equation related to $H$ reads

$$
\frac{\partial \sigma}{\partial t}=H \sigma, \quad \sigma_{\mid t=0}=\sigma_{0}
$$

and the solution

$$
\sigma(\cdot, \cdot, t)=\mathcal{F}_{B}^{-1}\left(\mathcal{F}_{B} \sigma_{0} \times \mathcal{F} K_{t}\right)
$$

coincides with the restriction on the diagonal $t_{1}=t_{2}$ of the solution (4.34).

In the case of color images, the system splits into three PDEs since the embedding space is of dimension 6 .

The algorithm (for a grey-level image) is the following one:

1. Compute the bivector $B_{1}$ encoding the tangent plane (formula (4.15)).

2. Construct an orthonormal frame field $\left(\nu_{11}, \nu_{12}, \nu_{21}, \nu_{22}\right)$ adapted to the $B_{1}$-plane and the $B_{2}=I_{4} B_{1}$-plane.

3. Compute the expression of the section $\sigma$ in the orthonormal frame field $\left(\nu_{11}, \nu_{12}, \nu_{21}, \nu_{22}\right)$.

4. In the orthonormal frame field $\left(\nu_{11}, \nu_{12}, \nu_{21}, \nu_{22}\right)$, the spinor Fourier transform splits into two usual complex Fourier transforms. 
5. Multiply the Fourier spectra with Gaussians of variance $t_{1}$ and $t_{2}$ for $t_{1}, t_{2} \in \mathbb{R}$.

6. Compute the inverse Fourier transforms.

7. Change the orthonormal frame field $\left(\nu_{11}, \nu_{12}, \nu_{21}, \nu_{22}\right)$ to the standard one.

We apply this algorithm on the grey level image and color image "Lake". In Fig. 2 are shown the original image, its projection on the tangent bundle and its projection on the normal bundle. Fig. 3 contains the results of the low-pass filtering interpreted as diffusion for different values of $t$. The diffusion acts as an Euclidean diffusion that preserves edges of the images. The interpretation in the case of a color image, Fig. 4 and 5, is more complicated since the color of the edge depends not only of the magnitude of the color variation but also of the colors that give the edge in the original image.

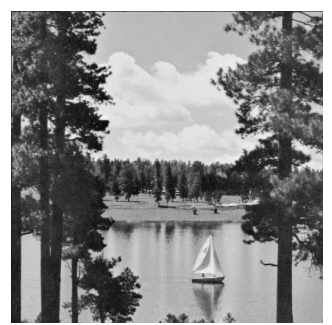

(a) Original

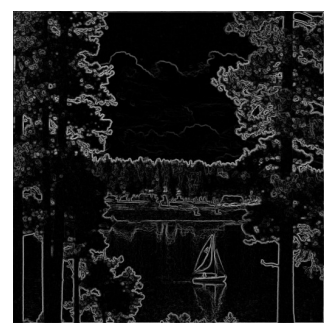

(b) Tangent part

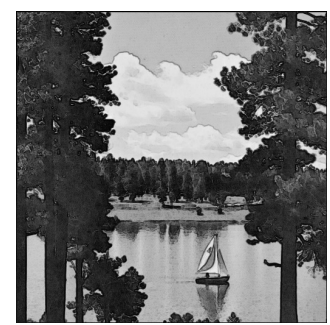

(c) Normal part

Figure 2. Decomposition of a grey-level image

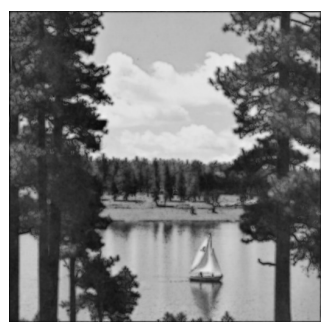

(a) $t=0.0001$

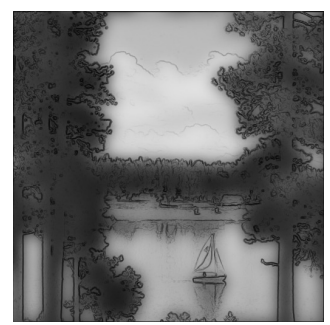

(b) $t=0.01$

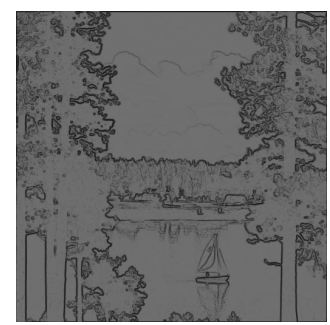

(c) $t \rightarrow \infty$

Figure 3. Diffusion on a grey-level image 


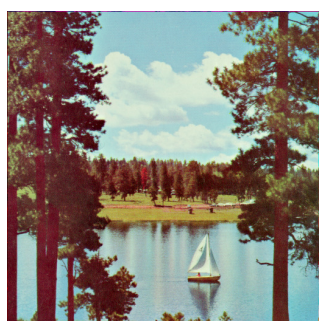

(a) Original

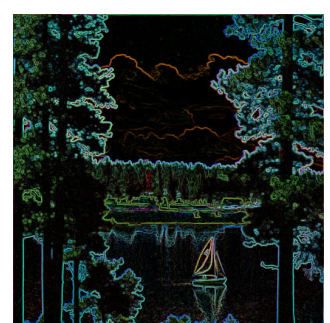

(b) Tangent part

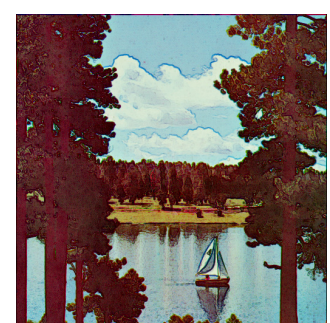

(c) Normal part

Figure 4. Decomposition of a color image

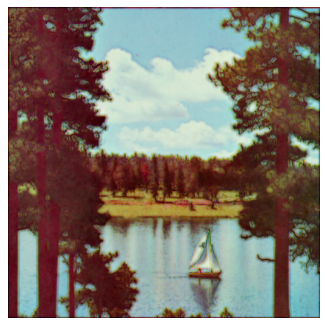

(a) $t=0.0001$

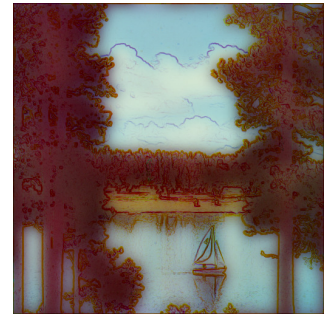

(b) $t=0.01$

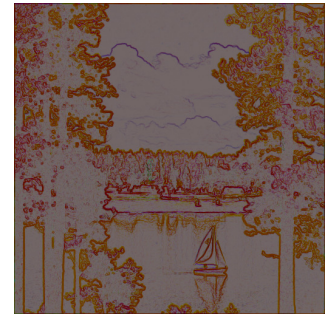

(c) $t \rightarrow \infty$

FiguRE 5. Diffusion on a color image

\section{Spinor Fourier Transform for Images using Spin Representations}

This section is devoted to the spin representation

$$
\zeta_{3}: \operatorname{Spin}(3) \longrightarrow \mathbb{C}(2)
$$

\subsection{Images as Sections of Spin Bunbles}

We consider a grey-level image

$$
f:\left(x_{1}, x_{2}\right) \in \Omega \longmapsto f\left(x_{1}, x_{2}\right)
$$

as a section

$$
\sigma_{f}:\left(x_{1}, x_{2}\right) \longmapsto\left(0, f\left(x_{1}, x_{2}\right)\right)
$$

of the bundle

$$
\Delta_{\zeta_{3}}(\Omega)=P_{S p i n}\left(E_{3}(\Omega)\right) \times_{\zeta_{3}} \mathbb{C}^{2}
$$

In the same way a color image

$$
f:\left(x_{1}, x_{2}\right) \in \Omega \longmapsto\left(f_{1}\left(x_{1}, x_{2}\right), f_{2}\left(x_{1}, x_{2}\right), f_{3}\left(x_{1}, x_{2}\right)\right)
$$

is considered as a section

$$
\sigma_{f}:\left(x_{1}, x_{2}\right) \longmapsto \sum_{k=1}^{3}\left(0, f_{k}\left(x_{1}, x_{2}\right)\right) \otimes e_{k}
$$


of the bundle

$$
\Delta_{\zeta_{3}}(\Omega) \otimes \mathbb{R}^{3}
$$

where $\left(e_{1}, e_{2}, e_{3}\right)$ is the standard basis of $\mathbb{R}^{3}$.

\subsection{Tangent Bundle and Bivector fields}

Recall that the bivector field $B=B_{1}$ chosen to treat the case of grey-level images is given by (4.15). When dealing with color images, we consider the following generalization of (4.15). The coefficients $\gamma_{i}, i=1,2,3$, are given by

$$
\gamma_{1}=\frac{1}{\delta} \quad \gamma_{2}=\frac{\sqrt{\sum_{k=1}^{3} f_{k, x_{2}}^{2}}}{\delta} \quad \gamma_{3}=-\frac{\sqrt{\sum_{k=1}^{3} f_{k, x_{1}}^{2}}}{\delta}
$$

where $f_{k, x_{i}}$ denotes the partial derivative of $f_{k}$ with respect to $x_{i}$ and

$$
\delta=\sqrt{1+\sum_{j=1}^{2} \sum_{k=1}^{3} f_{k, x_{j}}^{2}}
$$

The operator $B$ acting on the sections of $\Delta_{\zeta_{3}}(\Omega)$, where $\cdot$ denotes the Clifford multiplication, is represented by the $2 \times 2$ complex matrix

$$
B \cdot=\left(\begin{array}{cc}
i \gamma_{1} & -\gamma_{2}-i \gamma_{3} \\
\gamma_{2}-i \gamma_{3} & -i \gamma_{1}
\end{array}\right)
$$

Since $B^{2}=-1$ this operator has two eigenvalue fields $i$ and $-i$. Let $\nu_{i}^{B}$ and $\nu_{-i}^{B}$ be two corresponding unit eigenspinor fields given by

$$
\nu_{i}^{B}=\left(\begin{array}{l}
\frac{-\gamma_{3}+i \gamma_{2}}{\sqrt{2\left(1-\gamma_{1}\right)}} \\
\sqrt{\frac{1-\gamma_{1}}{2}}
\end{array}\right) \quad \nu_{-i}^{B}=\left(\begin{array}{l}
\frac{\gamma_{3}-i \gamma_{2}}{\sqrt{2\left(1+\gamma_{1}\right)}} \\
\sqrt{\frac{1+\gamma_{1}}{2}}
\end{array}\right)
$$

The spinor bundle $\Delta_{\zeta_{3}}(\Omega)$ splits into

$$
\Delta_{\zeta_{3}}(\Omega)=\Delta_{\zeta_{3}}^{(B,+)}(\Omega) \oplus \Delta_{\zeta_{3}}^{(B,-)}(\Omega)
$$

where

$$
\Delta_{\zeta_{3}}^{(B,+)}(\Omega)=\left\{\sigma \in \Delta_{\zeta_{3}}(\Omega), B \cdot \sigma=i \sigma\right\}
$$

and

$$
\Delta_{\zeta_{3}}^{(B,-)}(\Omega)=\left\{\sigma \in \Delta_{\zeta_{3}}(\Omega), B \cdot \sigma=-i \sigma\right\}
$$

The subbundles $\Delta_{\zeta_{3}}^{(B,+)}(\Omega)$ and $\Delta_{\zeta_{3}}^{(B,-)}(\Omega)$ are orthonormal and every section $\sigma$ of $\Delta_{\zeta_{3}}(\Omega)$ can be decomposed into $\sigma=\sigma_{+}^{B}+\sigma_{-}^{B}$ where

$$
\sigma_{+}^{B}=\frac{1}{2}(\sigma-i B \cdot \sigma) \in \Delta_{\zeta_{3}}^{(B,+)}(\Omega)
$$

and

$$
\sigma_{-}^{B}=\frac{1}{2}(\sigma+i B \cdot \sigma) \in \Delta_{\zeta_{3}}^{(B,-)}(\Omega)
$$

Using the orthonormal frame field $\left(\nu_{i}, \nu_{-i}\right)$, this can be written as

$$
\sigma=\sigma_{+} \nu_{i}^{B}+\sigma_{-} \nu_{-i}^{B}
$$




\subsection{Spinor Fourier Transform using Spin Representations}

Let us consider a grey-level image $f$ and the corresponding section $\sigma_{f}$ (see (5.3)). Although the spinor Fourier transform can be defined for an arbitrary bivector field $B$ that satisfies $B^{2}=-1$, we restrict ourselves to the case where $B$ is given by (4.15).

Definition 5.1 (Spinor Fourier transform for grey-level images - Spin Representations). With the above notations, the spinor Fourier transform of the section $\sigma_{f}$ is given by

$$
\mathcal{F}_{B} \sigma_{f}(A)=\int_{\mathbb{R}^{2}} \chi_{A, B\left(x_{1}, x_{2}\right)}\left(-x_{1},-x_{2}\right) \cdot \sigma_{f}\left(x_{1}, x_{2}\right) d x_{1} d x_{2}
$$

Recall that is this case the matrix $A$ is given by

$$
A=\left(\begin{array}{l}
u_{1} \\
u_{2}
\end{array}\right)
$$

Using the decomposition (5.17), one obtain

$$
\begin{gathered}
\chi_{A, B\left(x_{1}, x_{2}\right)}\left(-x_{1},-x_{2}\right) \cdot \sigma_{f}\left(x_{1}, x_{2}\right)=\left[\cos \left(u_{1} x_{1}+u_{2} x_{2}\right)-\right. \\
\left.\sin \left[\left(u_{1} x_{1}+u_{2} x_{2}\right)\right] B\right] \cdot\left(\sigma_{f+} \nu_{i}^{B}+\sigma_{f-} \nu_{-i}^{B}\right)\left(x_{1}, x_{2}\right)
\end{gathered}
$$

This means that

$$
\begin{gathered}
\chi_{A, B\left(x_{1}, x_{2}\right)}\left(-x_{1},-x_{2}\right) \cdot \sigma_{f}\left(x_{1}, x_{2}\right)= \\
{\left[\cos \left(u_{1} x_{1}+u_{2} x_{2}\right)-i \sin \left(u_{1} x_{1}+u_{2} x_{2}\right)\right] \sigma_{f+}\left(x_{1}, x_{2}\right) \nu_{i}^{B}\left(x_{1}, x_{2}\right)+} \\
{\left[\cos \left(u_{1} x_{1}+u_{2} x_{2}\right)+i \sin \left(u_{1} x_{1}+u_{2} x_{2}\right)\right] \sigma_{f-}\left(x_{1}, x_{2}\right) \nu_{-i}^{B}\left(x_{1}, x_{2}\right)}
\end{gathered}
$$

Hence, in the frame field $\left(\nu_{i}^{B}, \nu_{-i}^{B}\right)$, the spinor Fourier transform reads

$$
\mathcal{F}_{B} \sigma_{f}=\left({\widehat{\sigma_{f+}}}{\widehat{\sigma_{f-}}}^{-1}\right)
$$

where

$$
\widehat{\sigma_{f+}}(A)=\int_{\mathbb{R}^{2}} e^{-i\left(u_{1} x_{1}+u_{2} x_{2}\right)} \sigma_{f+}\left(x_{1}, x_{2}\right) d x_{1} d x_{2}
$$

and

$$
{\widehat{\sigma_{f-}}}^{-1}(A)=\int_{\mathbb{R}^{2}} e^{i\left(u_{1} x_{1}+u_{2} x_{2}\right)} \sigma_{f-}\left(x_{1}, x_{2}\right) d x_{1} d x_{2}
$$

This shows that in the frame field $\left(\nu_{i}^{B}, \nu_{-i}^{B}\right)$

$$
\begin{gathered}
\sigma_{f}\left(x_{1}, x_{2}\right)= \\
\left(\int_{\mathbb{R}^{2}} e^{i\left(u_{1} x_{1}+u_{2} x_{2}\right)} \widehat{\sigma_{f+}}\left(u_{1}, u_{2}\right) d u_{1} d u_{2}, \int_{\mathbb{R}^{2}} e^{-i\left(u_{1} x_{1}+u_{2} x_{2}\right)}{\widehat{\sigma_{f-}}}^{-1}\left(u_{1}, u_{2}\right) d u_{1} d u_{2}\right)
\end{gathered}
$$

Since we have chosen to define $\sigma_{f}$ by (5.3) this leads to an integral representation for $f$ :

$$
\begin{aligned}
& f\left(x_{1}, x_{2}\right)=\int_{\mathbb{R}^{2}}\left[\widehat{f_{+}}\left(u_{1}, u_{2}\right) e_{u_{1}, u_{2}}\left(x_{1}, x_{2}\right) \sqrt{\frac{1-\gamma_{1}}{2}}\right. \\
& \left.+\widehat{f}_{-}^{-1}\left(u_{1}, u_{2}\right) e_{-u_{1},-u_{2}}\left(x_{1}, x_{2}\right) \sqrt{\frac{1+\gamma_{1}}{2}}\right] d u_{1} d u_{2}
\end{aligned}
$$


where

$$
f_{+}=f \sqrt{\frac{1-\gamma_{1}}{2}} \quad f_{-}=f \sqrt{\frac{1+\gamma_{1}}{2}}
$$

and

$$
e_{u_{1}, u_{2}}\left(x_{1}, x_{2}\right)=\exp \left[i\left(u_{1} x_{1}+u_{2} x_{2}\right)\right]
$$

Let now $f$ be a color image and $\sigma_{f}$ be the corresponding section given by (5.6). Such an image can be decomposed as

$$
\begin{gathered}
f\left(x_{1}, x_{2}\right)=\int_{\mathbb{R}^{2}} \sum_{k=1}^{3}\left[\widehat{f_{k+}}\left(u_{1}, u_{2}\right) e_{u_{1}, u_{2}}\left(x_{1}, x_{2}\right) \sqrt{\frac{1-\gamma_{1}}{2}}\right. \\
\left.+{\widehat{f_{k-}}}^{-1}\left(u_{1}, u_{2}\right) e_{-u_{1},-u_{2}}\left(x_{1}, x_{2}\right) \sqrt{\frac{1+\gamma_{1}}{2}}\right] \otimes e_{k} d u_{1} d u_{2}
\end{gathered}
$$

where

$$
f_{k+}=f_{k} \sqrt{\frac{1-\gamma_{1}}{2}} \quad f_{k-}=f_{k} \sqrt{\frac{1+\gamma_{1}}{2}}
$$

\subsection{Applications}

The color image "Lake" is decomposed into $f=f_{+}+f_{-}$where (see formula $(5.27))$ :

$$
f_{+}=\sum_{k=1}^{3} f_{k+} \otimes e_{k} \quad f_{-}=\sum_{k=1}^{3} f_{k-} \otimes e_{k}
$$

Then, we perform a low-pass filtering by applying a Gaussian mask on each components of the Fourier transform of the section $\sigma_{f}$.

Fig. 6 shows the results of the filtering on the + component for different values of the variance. This component contains the edges of the color image and the low-pass filter makes them dissapear as the variance decreases.

Fig. 7 shows the results on the - component. This component contains more or less the homogeneous parts of the image. On each one of these parts, the filter acts as in an Euclidean space. The image tends to the mean color image as the variance decreases (in this case to a grey image).

\section{Conclusion}

We have shown how to define geometric Fourier transforms for grey-level and color image processing. The definitions rely on a spin generalization of the usual notion of characters involved in the abstract Fourier transform theory. Using spin geometry, we have proposed three possibilities to make act these characters on images. Applications to low-pass filterings show that these transforms preserve the structures of the images and treat color in a really non marginal way. One can envisage applications to color image compression or to the definition of generalized color Fourier descriptors in the spirit of $[26]$. 


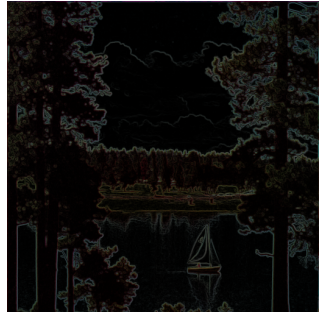

(a) + component

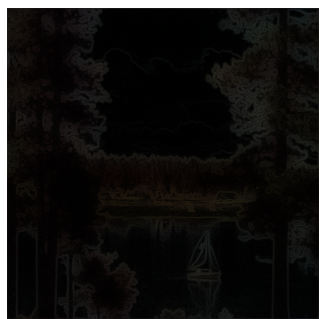

(d) Variance: 100

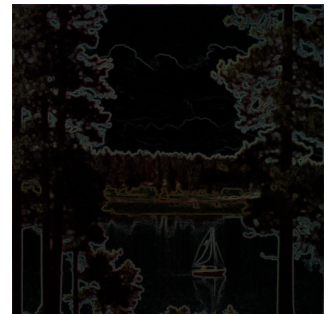

(b) Variance: 10000

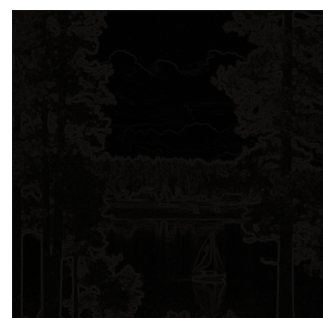

(e) Variance: 10

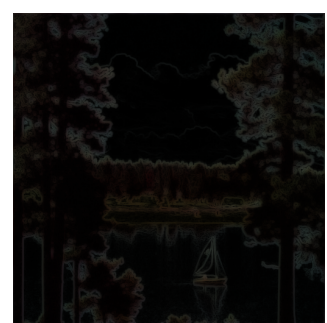

(c) Variance: 1000

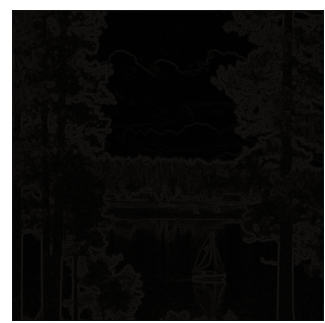

(f) Variance $\rightarrow 0$

Figure 6. Low-pass filter on the + component

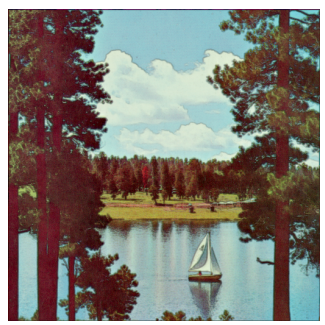

(a) Composante -

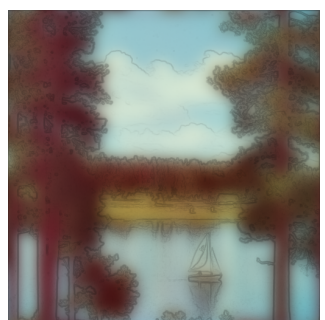

(d) Variance: 100

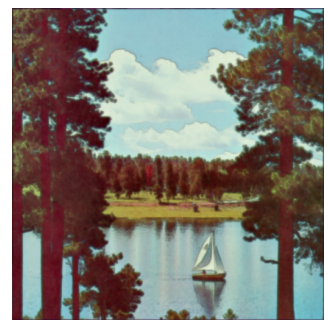

(b) Variance: 10000

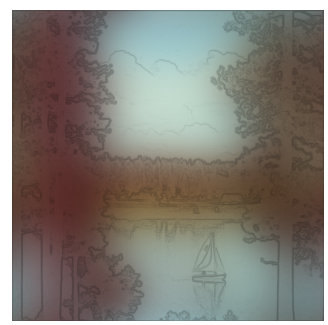

(e) Variance: 10

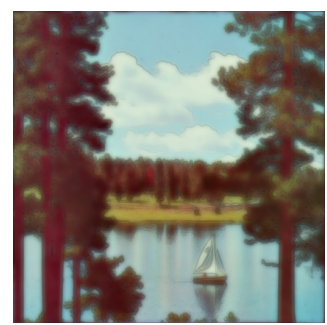

(c) Variance: 1000

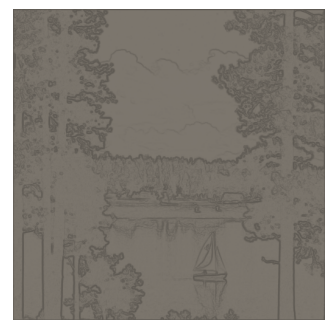

(f) Variance $\rightarrow 0$

Figure 7. Low-pass filter on the - component

\section{References}

[1] T. Batard and M. Berthier: Clifford algebra bundles to multidimensional image segmentation. Adv. Appl. Clifford Algebras 20, 2010, 3-4, pp. 489-516. 
[2] T. Batard and M. Berthier: Clifford Fourier transform and spinor representation of images. in Quaternion and Clifford Fourier Transforms and Wavelets (E. Hitzer and S.J. Sangwine Eds.), Trends in Mathematics (TIM), Birkhauser, Basel, 2012.

[3] T. Batard and M. Berthier: Spinor Fourier Transform for Image Processing. Preprint submitted.

[4] T. Batard, M. Berthier and C. Saint Jean: Clifford Fourier Transform for Color Image Processing. In Geometric Algebra Computing for Engineering and Computer Science. E. Bayro Corrochano and G. Scheueurmann Eds, Springer, 2010, pp.135-162.

[5] T. Batard, C. Saint Jean and M. Berthier: A Metric Approach to nD Images Edge Detection with Clifford Algebras. J Math Imaging Vis, 2009, vol. 33, pp. 296-312.

[6] F. Brackx, N. De Schepper and F. Sommen: The Two Dimensional Clifford Fourier Transform. J Math Imaging Vis, 2006, vol. 26, pp. 5-18.

[7] F. Brackx, N. De Schepper and F. Sommen: The Fourier transform in Clifford analysis. Adv. Imag. Elect. Phys. 156 (2008), pp. 55-203.

[8] R. Bujack, G. Scheueurmann and E. Hitzer: A general geometric Fourier transform. In 9th Int. Conf. on Clifford Algebras and their Applications in Mathematical Physics. K. Gürlebeck (ed.). Weimar, Germany, 15-20 July 2011. 19 pages.

[9] R. Bujack, G. Scheueurmann and E. Hitzer: A general geometric Fourier transform convolution theorem. To appear in Adv. Appl. Clifford Alg.

[10] T. Bülow: Hypercomplex Spectral Signal Representation for the Processing and Analysis of Images. Ph.D Thesis, Kiel, 1999.

[11] T. Bülow and G. Sommer: Hypercomplex signals - a novel extension of the analytic signal to the multidimensional case. IEEE Transactions on Signal Processing, 2001, Vol. 49, 11, pp. 2844-2852.

[12] C. Chevalley: The Algebraic Theory of Spinors and Clifford Algebras. Springer, New Edn., 1995.

[13] S. Dahlke, G. Kutyniok, G. Steidl and G. Teschke: Shearlet Coorbit Spaces and Associated Banach Frames. Appl. Comput. Harmon. Anal. 27 (2009) pp. 195-214.

[14] H. De Bie: Clifford algebras, Fourier transforms and quantum mechanics. To appear in Math. Methods Appl. Sci.

[15] H. De Bie and Y. Xu: On the Clifford Fourier transform. Int. Math. Res. Not. IMRN, 2011, No. 22, pp. 5123-5163.

[16] J. Ebling and G. Scheueurmann: Clifford Fourier Transform on Vector Fields. IEEE Transactions on Visualization and Computer Graphics, 2001, vol. 49 (11), pp. 2844-2852.

[17] T.A. Ell and S. Sangwine: Hypercomplex Fourier Transforms of Color Images IEEE Transactions on Image Processing, 2007, Vol. 16, 1, pp. 22-35.

[18] M. Felsberg: Low-level Image Processing with the Structure Multivector. Ph.D Thesis, Kiel, 2002.

[19] M. Felsberg and G. Sommer: The Monogenic Signal. IEEE Transactions on Signal Processing, 2001, vol. 49 (12), pp. 3136-3144. 
[20] T. Frankel: The Geometry of Physics - An Introduction. Revised Edition, Cambridge University Press.

[21] T. Friedrich: On the Spinor Representation of Surfaces in Euclidean 3-Space. Journal of Geometry and Physics, 28, 1998, pp. 143-157.

[22] D. Hestenes and G. Sobczyk: Clifford Algebra to Geometric Calculus. Reidel, Dordrecht, 1984.

[23] H.B. Lawson and M.-L. Michelson: Spin Geometry. Princeton University Press, Princeton, New Jersey, 1989.

[24] P. Lounesto: Clifford Algebras and Spinors. Second Edition, London Mathematical Society Lecture Note Series 286, Cambridge University Press, 2001.

[25] R. Lukac, K. N. Plataniotis, B. Smolka and A.N. Venetsanopoulos: Vector Operators for Color Image Zooming. IEEE Int. Symp. on Industrial Electronics, 2005.

[26] J. Mennesson, C. Saint Jean and L. Mascarilla: New Geometric Fourier Descriptors for Color Image Recognition. IEEE Int. Conf. on Image Processing, 2010 .

[27] M. Nakahara: Geometry, Topology and Physics. Sec. Edition, Graduate Student Series in Physics, Taylor and Francis (2003).

[28] M. Postnikov: Leçons de géométrie : groupes et algèbres de Lie. MIR, 1985.

[29] S. Sangwine and T.A. Ell: Hypercomplex Fourier Transform of Color Images. IEEE Int. Conf. on Image Processing, 2001, vol. 1, pp. 137-140.

[30] F. Smach, C. Lemaire, J.-P. Gauthier, J. Miteran and M. Atri: Generalized Fourier Descriptors with Applications to Objects Recognition in SVM Context. J Math Imaging Vis, 2008, vol. 30 (1), pp. 43-71.

[31] I.A. Taimanov: Two-dimensional Dirac operator and surface theory. Russian Mathematical Surveys, 61, 1, 2006, pp. 79-159.

[32] N.J. Vilenkin: Special Functions and the Theory of Group Representations. Translations of Mathematical Monographs, vol. 22. American Mathematical Society, Providence, Rhode Island (1968).

Michel Berthier

MIA Laboratory

La Rochelle University

Avenue Michel Crépeau

La Rochelle 17042

France

e-mail: michel.berthier@univ-lr.fr 